\section{Antineutrophil cytoplasmic antibodies and the eosinophilia myalgia syndrome}

The eosinophilia myalgia syndrome (EMS) is an inflammatory multisystem disease which emerged in epidemic form in 1989 and was linked to the ingestion of L-tryptophan. ${ }^{1}$ In the acute form of the EMS, severe myalgia and pronounced blood eosinophilia are accompanied by widespread organ involvement with the skin, lungs, serosal membranes, and the peripheral nervous system being involved most frequently. ${ }^{23}$ The chronic form of the EMS is characterised by progressive fascial and cutaneous fibrosis, leading to a clinical picture resembling eosinophilic fasciitis, accompanied by polyneuropathy, chronic fatigue, and psychological dysfunction. ${ }^{34}$

A spectrum of vascular lesions has been described including perivascular aggregation of mononuclear cells, intimal and medial thickening and fibrosis, ultrastructural alterations of the vascular endothelium, and frank vasculitis with mural infiltration by mononuclear cells. ${ }^{256}$ The last of these was found primarily in small arteries, veins and capillaries, and by immunofluorescence microscopy no deposition of immunoglobulins or complement components was found. 256

These histopathological features resemble those of the pauci-immune small vessel vasculitides, a group of diseases strongly associated with antineutrophil cytoplasmic antibodies (ANCA). ${ }^{7}$ In the March 1991 issue of this journal Cilursu and colleagues ${ }^{8}$ reported a patient with acute EMS and perinuclear ANCA (pANCA) with antimyeloperoxidase (MPO) specificity together with a small vessel vasculitis in skeletal muscle. This report prompted the question whether EMS also is an ANCA associated disease.

From a nationwide collection of EMS sera, we tested a random sample of 45 sera for ANCA. The sera were from 39 females (age 30-69 years) and six males (age 51-61 years) who fulfilled the diagnostic criteria of chronic EMS. ${ }^{4}$ ANCA testing was performed in compliance with the guidelines of the European ANCA Study Group with an indirect immunofluorescence test on ethanol fixed and formalin fixed granulocytes; antibody subspecificity was tested by specific enzyme linked immunosorbent assay

\section{LETTERS TO THE EDITOR}

(ELISA) using proteinase 3 (PR3), MPO, lactoferrin, lysozyme, elastase, and cathepsin $\mathrm{G}$ as antigens. ${ }^{9}$ For distinction between pANCA and antinuclear antibodies (ANA), sera were also examined on cultured human cells. ${ }^{10}$

We found none of the 45 sera to be positive for ANCA. Likewise all sera were negative by ELISA testing for the above mentioned antibody specificities. A number of sera produced fluorescent staining on the alcohol fixed granulocytes; however, this was attributable to ANA. In a Chang cell assay, 19 of 45 sera $(42 \%)$ tested positive for ANA.

The strongest clinical associations of ANCA have been shown to be that of antiPR3 with Wegener's granulomatosis (WG) and of anti-MPO with microscopic polyangiitis (MPA) and pauci-immune rapidly progressive glomerulonephritis (RPGN). These disorders have been categorised as the ANCA-associated vasculitides. In WG, $80 \%$ of patients are positive for cytoplasmic ANCA (cANCA); in generalised disease this is the case in more than $90 \%$, with antiPR3 being the most frequent antibody subspecificity (table). The association between pANCA or anti-MPO and MPA or pauci-immune RPGN is less strongapproximately $65 \%$ of MPA patients are antiMPO-positive. While anti-PR3 is highly specific for WG, anti-MPO has also been found at a low rate and in low titres in a number of other chronic inflammatory diseases. $^{7}$

A strong association of ANCA with secondary vasculitis has not yet been found. ${ }^{7}{ }^{10}$ The present results conform with this; notably, no serum showed anti-PR3 or anti-MPO reactivity. These results thus add to the evidence that anti-PR3 and anti-MPO are important markers for the classification and clinical diagnosis of primary systemic vasculitides, but not secondary vasculitides. The low prevalence of ANCA in the EMS makes it unlikely that ANCA have a pathogenetic role in this disorder.

ARMIN SCHNABEL
WOLFGANG L GROSS
Rheumatologische Poliklinik,
Medizinische Universität Lübeck,
D-23562 Lübeck, Germany

PETER A BERG RHEINHILD KLEIN

Medizinische Klinik II, Universität Tübingen, D-72076 Tübingen, Germany

HENDRIK LEHNERT Zentrum für Innere Medizin, Otto-von-Guericke-Universität Magdeburg, D-39120 Magdeburg, Germany

Correspondence to: Dr A Schnabel.

1 Carr L, Ruether E, Berg P A, Lehnert H Eosinophilia-myalgia syndrome in Germany: An epidemiologic review. Mayo Clin Proc 1994; 69: 620-5

2 Martin R W, Duffy J, Engel A G, et al. The clinical spectrum of the eosinophilia-myalg syndrome associated with ingestion. Ann Int Med 1990; 113: 124-34.

Clinical associations of antineutrophil cytoplasmic antibodies (ANCA)

\begin{tabular}{llccc}
\hline & $c A N C A$ & anti-PR3 & pANCA & anti-MPO \\
\hline Wegener's granulomatosis $(\mathrm{n}=445)$ & $358(80 \%)$ & $292(66 \%)$ & $5(1 \%)$ & $2(0 \cdot 4 \%)$ \\
Microscopic polyangitis $(\mathrm{n}=44)$ & $8(18 \%)$ & $3(7 \%)$ & $33(75 \%)$ & $28(64 \%)$ \\
Churg-Strauss syndrome $(\mathrm{n}=17)$ & $5(30 \%)$ & $5(30 \%)$ & $2(12 \%)$ & $1(6 \%)$ \\
EMS $(\mathrm{n}=45)$ & 0 & 0 & 0 & 0
\end{tabular}

cANCA = Cytoplasmic ANCA; pANCA = perinuclear ANCA; PR3 = proteinase $3 ; \quad$ MPO = myeloperoxidase; $\mathrm{EMS}=$ eosinophilia myalgia syndrome
pand
3 Kaufman L D, Gruber B L, Gregersen P K. Clinical follow-up and immunogenetic studies of 32 patients with eosinophiliamyalgia syndrome. Lancet 1991; 337: 1071-4.

4 Schnabel A, Reinhold-Keller E, Wolff $\mathrm{H} \mathrm{H}_{\text {, }}$ Gross W L. Fasziitis-SklerodermieEosinophilie-Syndrom durch Tryptophan. Dtsch Med Wochenschr 1991; 116: 1180-5.

5 Smith S A, Roelofs R I, Gertner E. Microangiopathy in the eosinophilia-myalgia Myndrome. $\mathcal{f}$ Rheumatol 1990; 17: 1544-50.

6 Tazelaar H D, Myers J L, Strickler J G Tazelaar H D, Myers J L, Strickler J G,
Colby T V, Duffy J. Tryptophan-induced lung disease: an immunophenotypic, immunofluorescent, and electron microscopic study. Mod Pathol 1993; 6: 56-60.

7 Gross W L, Schmitt H H, Cisernok E. ANCA and associated diseases: immunodiagnostic and pathogenetic aspects. Clin Exp Immunol 1993; 91: 1-12.

8 Cilursu A M, Goeken J, Olson R R. Detection of antineutrophil cytoplasmic antibody in a patient with L-tryptophan induced eosinophilia-myalgia philia-myalgia syndrome [correction in Ann Rheum Dis 1992,
1991; 50: 817-9.

9 Hagen E C, Andrassy K, Csernok E, et al. The value of indirect immunofluorescence and value of indirect immunofluorescence and $A$ report on the first phase of an international cooperative study on the standardization of ANCA assays. EEC/BCR Group for ANCA Assay Standardization. $\mathcal{f}$ Immunol Methods 1993; 159: 1-16.

10 Schnabel A, Csernok E, Isenberg D A Mrowka C, Gross W L. Anti-neutrophil cytoplasmic antibodies-prevalence, specifcities, and clinical significance. Arthritis Rheum. In press.

\section{Silent myocardial infarction in Wegener's granulomatosis}

At postmortem, patients with Wegener's granulomatosis (WG) frequently show a clinically overlooked and diffuse disease process. Cardiologically, many such patients have been asymptomatic but present histological findings of specific heart involvement. We report two cases of silent myocardial infarction related to WG-a feature which, to our knowledge, has not been reported in clinical series.

Patient 1. A 42 year old man was admitted to hospital because of severe vasculitis flare. He had an eight month history of arthritis and lower limb dysaesthesia. Examination showed an acutely ill patient with a $39^{\circ} \mathrm{C}$ fever. He had oral ulcers, haemorrhagic gingival hyperplasia, bilateral haemorrhagic nasal discharge with crusts, diffuse necrotic purpura and black discolouration of fingers and toes. Ankle jerks were absent. Standard blood tests showed: leucocyte count $9.6 \times 10^{9} / 1$; serum creatinine $112 \mu \mathrm{mol} / \mathrm{l}$; C reactive protein $278 \mathrm{mg} / \mathrm{l}$; fibrin $12 \mathrm{~g} /$; total creatine kinase $(\mathrm{CK})$ concentration $1102 \mathrm{U}$ (normal range 15-90 $\mathrm{U}$ ) with $\mathrm{MB}$ isoenzyme 130 U. Cholesterol, triglyceride and blood glucose values were within the normal range. Microscopic haematuria was present at 15/HPF. The electrocardiogram showed ST segment elevation and loss of $R$ waves in leads V1, V2, and V3. Silent anterior myocardial infarction was diagnosed and WG was suspected. It was confirmed by: a) antineutrophil cytoplasmic antibodies (diffuse cytoplasmic fluorescence) in the serum; b) pansinusitis demonstrated by computed tomography scan; c) multineuritis on electrophysiological examination; d) necrotising vasculitis on skin biopsy. Lupus anticoagulant and anticardiolipin antibodies were absent. Antithrombin III, protein C and 
protein $S$ plasma concentrations were normal. Initial treatment comprised intravenous administration of methylprednisolone, cyclophosphamide and sodium heparinate. Other drugs included diltiazem, dinitrosorbide, and enalapril. The short term course was uneventful. At day 14, the patient had a sudden rupture of the spleen Splenectomy was performed. Histological analysis of the spleen showed widespread necrotising vasculitis with haemorrhagic infarction. At day 30, coronarography was normal. At month 24, the patient had no active sign of WG nor any recurrence of myocardial ischaemia.

Patient 2. A 41 year old woman was admitted for suspected systemic vasculitis She had a two year history of crusted rhinitis with relapsing sinusitis and reported productive cough with bloody sputum. A chest radiograph showed disseminated excavated pulmonary nodules. Fibroscopy disclosed a pseudotumoural aspect of the bronchial mucosa. Microscopic examination of a bronchial specimen showed giant cell granuloma. Treatment against tuberculosis was started with four drugs. Because distressing neurological signs appeared the patient was transferred to our department. Physical examination showed a febrile, disoriented, acutely ill woman with nasa obstruction, bilateral ear chondritis, diplopia, right upper eyelid ptosis, dysaesthesia and paresia in the left foot, and absent ipsilateral ankle jerk. Chest auscultation was normal. Standard blood tests showed: leucocyte count $16.7 \times 10^{9} / \mathrm{l}$; creatinine $52 \mu \mathrm{mol} / \mathrm{l}$; C reactive protein $252 \mathrm{mg} / \mathrm{l}$; fibrin $10 \mathrm{~g} / \mathrm{l}$; total CK concentration $347 \mathrm{U}$ with $\mathrm{MB}$ isoenzyme $179 \mathrm{U}$. Cholesterol, triglycerides and blood glucose values were within the normal range. Lowgrade haematuria was present at 10/HPF. The electrocardiogram showed ST segment and $\mathrm{T}$ wave elevation in leads V4 and V5, with loss of $R$ waves in the anterior leads. Echocardiography showed septa akinesia and diffuse hypocontractility withou dilatation of the left ventricle. Silent acute anteroseptal myocardial infarction was diagnosed. A diagnosis of severe WG was further supported by: a) antineutrophil cytoplasmic antibodies (diffuse cytoplasmic fluorescence) in the serum; b) mononeuritis multiplex on electromyographic study; c) multiple bilateral infarcts of the white matte on cerebral magnetic resonance imaging; d) typical granulomatous necrotising vasculitis on nasal biopsy. Tests for antiphospholipid antibodies were negative. Treatment included intravenous pulses of high-dose prednisolone followed by oral prednisone, monthly intravenous cyclophosphamide, and sodium heparinate. All symptoms remitted rapidly, except for mononeuritis multiplex that progressively deteriorated over a period of months. After eight cycles, cyclophosphamide was changed for daily oral etoposide which has been considered to be effective in neuropathy. ${ }^{1}$ At month 18 , relapse of sinusitis and pulmonary nodules required increased prednisone dosage and etoposide was stopped in favour of weekly methotrexate. At month 22, the patient had no active sign of WG nor any recurrence of myocardial ischaemia.

Because Wegener's granulomatosis was once a uniformly fatal disease, before successful use of cyclophosphamide therapy, early reports were only of postmortem findings. Walton, reviewing 54 cases, found myocardial granulomata in six and focal coronaritis in 15; death was retrospectively attributed to myocardial infarction in two cases. ${ }^{2} \mathrm{~A}$ more recent comprehensive review reported the most common pathological findings as pericarditis in $50 \%$, focal myocarditis in $25 \%$, coronary arteritis in $50 \%$, and myocardial infarction in $11 \%$ of WG postmortem cases in which the heart was examined. ${ }^{3}$

Such high prevalence contrasts sharply with clinical data collected in the cyclophosphamide era. Only $10(6 \%)$ of 158 WG patients had detectable heart involvement in the series reported by Hoffman et al. ${ }^{4}$ All had specific pericarditis and no myocardial ischaemia was reported. ${ }^{5}$ Heart involvement was not mentioned among the causes of death. In contrast, there have been several published reports of WG with myocardial involvement-heart muscle disease, heart block and supraventricular tachycardia, and cardiac mass. ${ }^{5-7}$

Our two patients had myocardial infarction clearly related to WG because: a) myocardial infarction was novel, with no angina in the past; b) myocardial infarction accompanied severe generalised WG flare, c) the extent of clinical systemic vasculitis was unusually diffuse (distal limb necrosis and splenic infarction in patient 1 and cerebral vasculitis in patient 2 ); d) atheroma could be rejected as the cause of myocardial infarction because of normal coronarography in patient 1 and absence of any risk factor in patient 2; and e) no cardiac ischaemic event was observed during a two year follow up under immunosuppressive therapy.

In the literature, we could find only two reports of myocardial infarction recognised in alive WG patients. Gatenby et al report the fulminant course of WG in a 28 year old man who died from painful and massive myo- cardial infarction. Pathological examination of the heart showed vasculitis with fibrinoid necrosis of the media and partial occlusion of the lumen by pale antemortem thrombus in all coronary vessels. ${ }^{8}$ In the other case reported in the literature, an embolic mechanism rather than vasculitis could account for the symptomatic myocardial ischaemia. ${ }^{9}$

Wegener's granulomatosis is defined as a small vessel vasculitis. The severity of the condition, and occasional associated death, might be related to the progression of vasculitis and thrombosis to larger sized vessels. ${ }^{10}$ Coronary wall inflammation and formation of lumen blood clot are two mutually non-exclusive processes that may have been responsible for myocardial ischaemia in our two patients.

In practical terms, cardiac enzymes and electrocardiography should be repeatedly monitored in a patient with WG flare.

THOMAS PAPO JEAN-CHARIES PIETTE RACHID LARAKI OLIVIER BLETRY DU LE THI HUONG PIERRE GODEAU Department of Internal Medicine, Hôpital Pitié-Salpêtrière, Paris, France

Correspondence to: Dr Papo, Internal Medicine Unit, Hôpital Pitié-Salpêtrière, 83 Boulevard de l'Hôpital, 75651 Paris cedex 13, France.

1 D'Cruz D, Payne H, Timothy A, Hughes G R V. Response of cyclophosphamide-resistant Wegener's granulomatosis to etoposide. Lancet 1992; 340: 425-6.

2 Walton E W. Giant-cell granuloma of the respiratory tract (Wegener's granulomatosis). respiratory tract (Wegen $1958 ; 2$ : 265-70.

3 Forstot J Z, Overlie P A, Neufeld G K, Harmon C E, Forstot S L. Cardiac complications of Wegener granulomatosis: a
case report of complete heart block and case report of complete heart block and
review of the literature. Semin Arthritis Rheum review of the literatur

4 Hoffman G S, Kerr G S, Leavitt R Y, et al. Wegener granulomatosis: an analysis of 158 patients. Ann Int Med 1992; 116: 488-98.

5 Hoffman G S, Fauci A S. Wegener granulomatosis (in response). Ann Int Med 1992; 117: 620-1.

6 Schiavone W A, Ahmad M, Ockner S A. Unusual cardiac complications of Wegener's granulomatosis. Chest 1985; 88: 745-8.

7 Kosovsky P A, Ehlers K H, Rafal R B, Williams W M, O'Loughlin J E, Markisz J A. WR imaging of cardiac mass in Wegener Mranulomatosis. f Comput Assist Tomogr 1991; granulomatosis.

8 Gatenby P A, Lytton D G, Bulteau V G, O'Reilly B, Basten A. Myocardial infarction in Wegener's granulomatosis. Aust NZ F Med 1976; 6: 336-40.

9 Gerbracht D D, Savage R W, Scharff N. Reversible valvulitis in Wegener's granulomatosis. Chest 1987; 92: 182-3.

10 McCrea P C, Childers R W. Two unusual cases of giant cell myocarditis associated with mitral valve stenosis and with Wegener's syndrome. Br Heart $¥$ 1964; 26: 490-8. 